\title{
Experiencia quirúrgica inicial en pacientes con COVID-19
}

\section{Surgical initial experience in patients with COVID-19}

\author{
Carlos O. Aguilar-Garduño ${ }^{1}$, José C. Aguilar-Garduño², Ana K. Barreto-Rodríguez', \\ Vanessa Ortiz-Higareda ${ }^{1}$, Eduardo Ferat-Osorio ${ }^{3}$ y José L. Martínez-Ordaz ${ }^{3 *}$
}

${ }^{1}$ Servicio de Gastrocirugía, Hospital de Especialidades del Centro Médico Nacional Siglo XXI, Instituto Mexicano del Seguro Social (IMSS); ${ }^{2}$ Facultad Mexicana de Medicina, Universidad La Salle; ${ }^{3}$ Dirección de Educación e Investigación en Salud, Hospital de Especialidades del Centro Médico Nacional Siglo XXI, IMSS. Ciudad de México, México

\section{Resumen}

Antecedentes: La pandemia de COVID-19 ha ocasionado que los servicios de cirugía y de salud en todo el mundo tengan que reorganizarse y planear para poder brindar la mejor atención a los pacientes, con la protección necesaria para el personal de salud. Algunos de estos pacientes requerirán tratamiento quirúrgico, ya sea electivo o de urgencia. Objetivo: Reportar la experiencia inicial en el manejo de pacientes con COVID-19 que ameritaron tratamiento quirúrgico por los servicios de cirugía de un hospital de referencia. Método: Revisión de los protocolos quirúrgicos, equipo de protección personal usado por los equipos quirúrgicos y resultados del tratamiento de 42 pacientes sometidos a cirugía en un periodo de 4 meses. Resultados: Fueron intervenidos 42 pacientes con COVID-19. Treinta pacientes tenían diagnóstico de infección por SARSCoV-2 y en 12 casos el diagnóstico fue clínico y por imagen. Las cirugías más frecuentes fueron traqueostomía en 16 pacientes (38\%) y laparotomías exploradoras en 8 pacientes (19\%). La mediana de estancia posoperatoria fue de 17 días y la mortalidad durante los primeros 30 días fue del $26 \%$. Conclusiones: Es necesaria la reorganización de los departamentos quirúrgicos y del hospital para poder atender adecuadamente a los pacientes con COVID-19 y proteger al personal de salud. Los pacientes pueden presentan patologías que requieran tratamiento quirúrgico. Relacionado con la infección y la mayor frecuencia de comorbilidad, la mortalidad de estos pacientes es elevada.

Palabras clave: COVID-19. SARS-CoV-2. Cirugía.

\section{Abstract}

Introduction: the COVID-19 pandemic has caused a reorganization of hospital and general surgery departments worldwide to assure the best medical and surgical treatment of patients with this disease and protection of the health-related personnel. Some of them will require surgical treatment either elective or urgent. Objective: report the initial experience in the management of patients with COVID-19 in a third level hospital. Material and Methods: a review of the surgical protocols, personal protection equipment used by the surgical teams, and results of the treatment of forty-two patients submitted to surgery. Results: During four months (April-July 2020) forty-two patients with suspicion or confirmed infection of SARS-CoV2 underwent surgical treatment. The most common surgery was tracheostomy in 16 patients (38\%) followed by exploratory laparotomy in 8 patients (19\%). The median postoperative stay was 17 days and the thirty-day postoperative mortality rate was $26 \%$. Conclusions: reorganization of the general surgery department and the hospital, favors adequate management and treatment

\section{Correspondencia:}

*José L. Martínez-Ordaz

Avda. Cuauhtémoc, 330

Col. Doctores, Del. Cuauhtémoc

Fecha de recepción: 03-09-2020

C.P. 06720 , Ciudad de México, México

E-mail: jIm01968@gmail.com

0009-7411/@ 2020 Academia Mexicana de Cirugía. Publicado por Permanyer. Este es un artículo open access bajo la licencia CC BY-NC-ND (http://creativecommons.org/licenses/by-nc-nd/4.0/).
Cir Cir. 2021;89(2):183-188

Contents available at PubMed www.cirugiaycirujanos.com
Fecha de aceptación: 27-11-2020

DOI: $10.24875 / C I R U .20000972$ 
of patients with COVID-19 and protection to the health-related personnel. Due to the usual co-existence of comorbidities and pulmonary complications the postoperative mortality of these patients is high.

Key words: COVID-19. SARS-CoV-2. Surgery.

\section{Introducción}

En diciembre de 2019 se comenzó a reportar la presencia de neumonías virales en la ciudad de Wuhan, China. Tras el estudio de casos, fue identificado un nuevo virus, el SARS-CoV-2 y fue descrita la enfermedad COVID-19. El primer caso en México fue confirmado el 28 de febrero de 2020. Fue declarada pandemia por la Organización Mundial de la Salud el 9 de marzo del 2020. Al momento de escribir este reporte, hay más de 647,000 casos confirmados y 69,000 muertes relacionadas con esta enfermedad en el país. Como consecuencia, fueron diseñadas estrategias en el sector salud y hospitalarias para la atención de los pacientes con la enfermedad, separarlos de los pacientes sin ella, y contener la diseminación tanto en otras poblaciones como en los trabajadores del sector salud.

Los pacientes con patologías quirúrgicas agudas seguirán presentándose y algunas de ellas pueden poner en peligro la vida y requerir cirugía urgente. Las indicaciones para cirugía en pacientes con COVID-19 son las mismas que en la población general'. La meta es otorgar un tratamiento adecuado y oportuno a los pacientes, optimizando el uso de los recursos hospitalarios (camas, terapia intensiva, equipos de protección personal (EPP), ventiladores, entre otros). No hay sustituto para el criterio quirúrgico, la cirugía debe ser realizada si al diferirla puede ocasionar daño al paciente, mayor estancia o readmisión hospitalaria o el tratamiento médico ha fallado $0^{2,3}$.

El objetivo de este trabajo es reportar la experiencia de pacientes con COVID-19 que fueron sometidos a tratamiento quirúrgico en un hospital de tercer nivel durante un periodo de cuatro meses.

\section{Pacientes $Y$ metodos}

Durante un periodo de 4 meses ( 1 de abril al 31de julio de 2020) fueron recabados todos los pacientes que fueron intervenidos quirúrgicamente con el diagnóstico o sospecha de infección por SARS-CoV-2 o sospechosos de esta infección en un hospital de tercer nivel. La infección debía ser corroborada por la determinación de PCR (prueba de reacción de polimerasa) o sospechada por cuadro clínico y cambios compatibles demostrados por tomografía computada de tórax.

Para la atención de los pacientes con COVID-19 se diferenciaron dos áreas de atención de urgencias separadas e independientes entre sí, la usada regularmente y una segunda donde son dirigidos los pacientes con datos de infección de vías aéreas o sospechosos para presentar COVID-19 (triage respiratorio). Los preparativos hospitalarios también incluyeron desarrollar un área de hospitalización y terapia intensiva COVID-19, con ventiladores y equipos de protección personal (EPP) para el personal de salud encargado del manejo de los pacientes, el cual recibió capacitación para la colocación y retiro de éste. Conforme a la distribución del hospital, fueron destinados 4 quirófanos exclusivos para los pacientes con COVID-19. En el resto del hospital las cirugías electivas fueron jerarquizadas por servicio, así se disminuyó el número de cirugías de 596 electivas y 255 urgentes en marzo a 83 cirugías electivas y 117 cirugías urgentes en promedio en estos 4 meses. La consulta externa disminuyó en el mismo periodo de 13,700 a 5980 consultas mensuales en promedio.

Del grupo de pacientes fueron obtenidos los datos relacionados con COVID-19, datos demográficos, presencia de comorbilidades, indicación quirúrgica, tipo de intervención quirúrgica (urgente o electiva y cirugía mayor o menor), procedimiento quirúrgico realizado, evolución posoperatoria y desenlace (egreso o muerte).

Para el ingreso del paciente con COVID-19 a quirófano existe una ruta exclusiva, demarcada y señalizada, con entrada directa a las salas de operación. Todo el equipo quirúrgico participante utilizó EPP consistente en bata impermeable, gorro, lentes con protección lateral ("goggles"), respiradores N95, botas y doble par de guantes de látex. Por encima de ellos se colocaron, previo lavado con alcohol gel de los guantes, nueva bata quirúrgica y otros pares de guantes con técnica estéril.

\section{Resultados}

En un periodo de cuatro meses fueron ingresado al área COVID un total de 699 pacientes sospechosos 
Tabla 1. Características demográficas, comorbilidades y manifestaciones clínicas en 42 pacientes con COVID-19 o sospechosos de infección por SARS-CoV2

\begin{tabular}{|c|c|c|}
\hline & $\mathbf{N}^{\circ}$ & Porcentaje \% \\
\hline \multicolumn{3}{|l|}{ Género } \\
\hline Masculino & 19 & $45 \%$ \\
\hline Femenino & 23 & $55 \%$ \\
\hline \multicolumn{3}{|l|}{ Comorbilidades } \\
\hline Hipertensión arterial & 21 & $50 \%$ \\
\hline Diabetes mellitus & 18 & $43 \%$ \\
\hline Obesidad & 17 & $40 \%$ \\
\hline Enfermedad renal crónica & 2 & $8 \%$ \\
\hline Otras & 1 & $4 \%$ \\
\hline \multicolumn{3}{|l|}{ PCR COVID 19} \\
\hline Positivo & 30 & $71 \%$ \\
\hline Negativo & 12 & $29 \%$ \\
\hline \multicolumn{3}{|l|}{ TAC compatible COVID19 } \\
\hline Positivo & 37 & $88 \%$ \\
\hline Negativo & 5 & $12 \%$ \\
\hline \multicolumn{3}{|l|}{ Signos y Síntomas } \\
\hline Fiebre & 35 & $83 \%$ \\
\hline Mialgias y artralgias & 27 & $64 \%$ \\
\hline Tos seca & 22 & $52 \%$ \\
\hline Disnea & 20 & $48 \%$ \\
\hline Dolor abdominal & 11 & $26 \%$ \\
\hline Cefalea & 9 & $21 \%$ \\
\hline
\end{tabular}

DM tipo2: Diabetes mellitus tipo2, HAS: hipertensión arterial sistémica,

ERC: enfermedad renal crónica, TAC: Tomografía axial computada de tórax, n: Número, PCR: polymerase chain reaction.

o confirmados por SARS-CoV-2. De estos, 42 pacientes $(6 \%)$ requirieron tratamiento quirúrgico durante su internamiento.

Fueron 19 hombres y 23 mujeres, con una mediana de edad de 61 años (IQ 49.7-74). Presentaban alguna comorbilidad 31 pacientes (74\%), siendo las más comunes, hipertensión arterial en $21(50 \%)$, diabetes mellitus en $18(43 \%)$ y obesidad en $17(40 \%)$ pacientes (Tabla 1).

En todos los casos, la infección por SARS-CoV-2 fue sospechada o confirmada previo a la cirugía. En 30 pacientes $(71 \%)$ la infección fue demostrada por la prueba de PCR para SARS-CoV-2. En los 12 pacientes restantes fue sospechada por imagen radiológica y tomográfica compatible con COVID-19 (Fig. 1). Los síntomas más frecuentes relacionados con COVID-19 fueron fiebre en 35 pacientes (83\%), tos seca en 20 (48\%), dificultad respiratoria 22 (52\%), mialgias y artralgias 27 (64\%) (Tabla 1).

Las cirugías fueron consideradas urgentes en 24 pacientes (58\%). En total fueron clasificadas como cirugía mayor en 22 pacientes (52\%) y en 20 como cirugía menor (48\%). La indicación más frecuente de

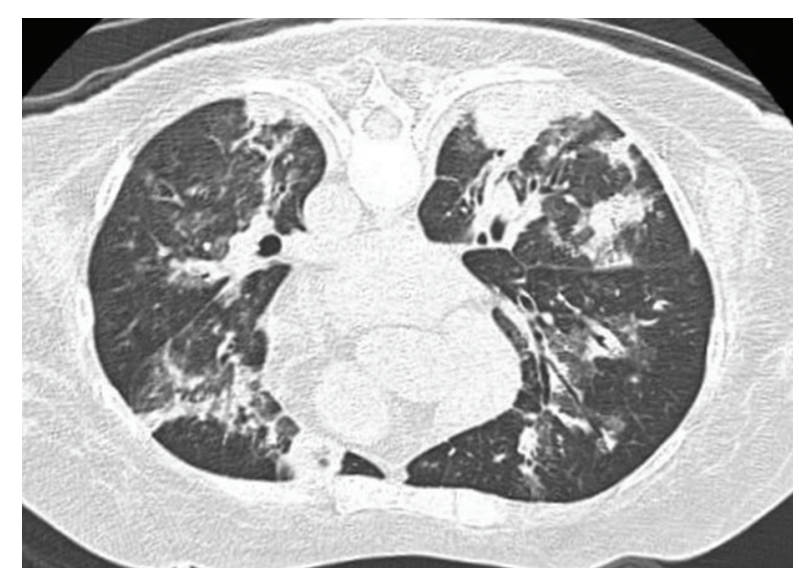

Figura 1. Imagen de tomografía computada de tórax donde se observa parénquima pulmonar con presencia de múltiples áreas difusas de consolidación de distribución periférica y subpleural, multisegmentaria, asociada a patrón de vidrio deslustrado adyacente, asi como atelectasias subsegmentarias en ambas bases pulmonares. Hallazgos de imagen comúnmente reportados de neumonía por COVID-19.

tratamiento quirúrgico fue intubación prolongada con incapacidad para el retiro del apoyo mecánico ventilatorio en 16 pacientes (38\%) por lo que la cirugía más frecuentemente realizada fue traqueostomía. La mediana de día de intubación en la cual se realizó el procedimiento fue de 22.5 días (IQ 19-24). El resto de las indicaciones y procedimientos quirúrgicos realizados se encuentran en la tabla 2 y tabla 3 respectivamene. Todos los procedimientos se realizaron con anestesia general.

La mediana de estancia posoperatoria fue de 17 días (IQ 12-41). No hubo reintervenciones quirúrgicas no programadas. Fallecieron durante los primeros 30 días posoperatorios 11 pacientes (26\%). Las causas de muerte fueron complicaciones respiratorias en 8 pacientes (19\%), sepsis abdominal en $2(4.7 \%)$ y hemorragia posoperatoria en 1 (4\%).

\section{Discusión}

Existe la posibilidad de pacientes con COVID-19 requieran cirugía ya sea por enfermedades concomitantes o complicaciones derivadas de esta enfermedad. Este trabajo presenta la experiencia de los pacientes con COVID-19 o sospechosos de infección por SARS-CoV-2 en fueron intervenidos quirúrgicamente en un hospital de tercer nivel. Para determinar la necesidad de la intervención quirúrgica, éstas pueden ser jerarquizadas desde aquellas que deben ser resueltas inmediatamente hasta un periodo de dos semanas, a las que pueden ser realizadas en 8 o más 
Tabla 2. Indicaciones quirúrgicas en cuarenta y dos pacientes con diagnóstico o sospechosos de tener COVID-19

\begin{tabular}{lcc}
\hline Diagnostico Preoperatorio & $\mathbf{N}^{\circ}$ & Porcentaje \\
\hline Intubación prolongada & 16 & $38 \%$ \\
Insuficiencia arterial aguda & 4 & $9.5 \%$ \\
Enfermedad diverticular complicada & 3 & $7.1 \%$ \\
Aneurisma cerebral roto & 2 & $4.7 \%$ \\
Absceso subfrénico derecho & 2 & $4.7 \%$ \\
Colecistitis aguda & 2 & $4.7 \%$ \\
Fascitis necrotizante & 2 & $4.7 \%$ \\
Mucormicosis & 2 & $4.7 \%$ \\
Gangrena de Fournier & 2 & $4.7 \%$ \\
Cuerpo extraño en esófago & 1 & $2.3 \%$ \\
Apendicitis aguda & 1 & $2.3 \%$ \\
Embarazo de 23.1 SDG + Sufrimiento fetal & 1 & $2.3 \%$ \\
Endoftalmitis endógena de ojo izquierdo & 1 & $2.3 \%$ \\
Hematuria macroscópica & 1 & $2.3 \%$ \\
Intolerancia a la vía oral & 1 & $2.3 \%$ \\
Hidrocefalia comunicante & 1 & $2.3 \%$ \\
\hline
\end{tabular}

Tabla 3. Intervencionaes quirúrgicas en cuarenta y dos pacientes con diagnóstico o sospechosos de tener COVID-19

\begin{tabular}{|c|c|c|}
\hline Cirugía realizada & $\mathrm{N}^{\circ}(\%)$ & Complicaciones \\
\hline Traqueostomía & $16(38 \%)$ & NO \\
\hline LAPE & $8(19 \%)$ & $\mathrm{SI}(2)^{*}$ \\
\hline Exploración arterial & $4(9.5 \%)$ & NO \\
\hline Lavado quirúrgico & $4(9.5 \%)$ & NO \\
\hline Craneotomía & $3(7 \%)$ & NO \\
\hline Cesárea & $1(4.1 \%)$ & $\mathrm{SI}^{* *}$ \\
\hline Otras ${ }^{\star * \star}$ & $7(29.16 \%)$ & NO \\
\hline
\end{tabular}

semanas $^{3,4}$. En los pacientes con COVID-19, las cirugías que no se consideren urgentes deberán ser pospuestas $^{5}$. El incremento el riesgo que implica la infección por SARS-CoV-2, debe evaluarse con el riesgo de retrasar el tratamiento quirúrgico; así como tomar en cuenta individualmente los factores de mal pronóstico encontrados en los pacientes que es más frecuente que ameriten hospitalización, como edad $>50$ años y, la presencia de comorbilidades como obesidad, diabetes mellitus, complicaciones pulmonares y cardiopatía. Las medidas de protección antes, durante y posteriores al tratamiento quirúrgico tienen que ser mayores e individualizadas durante esta pandemia.No hay sustituto para el criterio quirúrgico².

Además, los pacientes que ingresan de manera ambulatoria que requieren cirugía electiva considerada como esencial deben ser monitorizados buscando fiebre, síntomas respiratorios e historia de contactos con casos sospechosos ${ }^{6}$. Derivado de que la cantidad de las pruebas es limitada y el tiempo de resultado de ésta no es práctico, en caso de duda ante la presencia de situaciones urgentes, todo paciente debe ser manejado como sospechoso de presentar la enfermedad ${ }^{1,7}$.

Idealmente los pacientes con COVID-19 deben estar en habitaciones aisladas o asignados como cohorte con otros pacientes positivos. El personal incluido en el manejo de estos pacientes no debe circular por el resto del hospital'.

De contar con ellas, las salas de operaciones deben ser con presión negativa y exclusivas para pacientes positivos o sospechosos ${ }^{6,8}$. En nuestro hospital fue posible destinar dos bloques de quirófanos. En caso de no tener la posibilidad de hacer esto, idealmente el paciente debe ser programado como el último del día ${ }^{3}$. El quirófano destinado para pacientes con COVID-19 debe ser el primero desde la entrada al bloque de quirófano para evitar la contaminación del resto ${ }^{7}$. El paciente entra directamente sin permanecer en sala preoperatoria. Dentro de quirófano es conveniente el menor instrumental quirúrgico y mobiliario específico para la cirugía posible ${ }^{9}$.

Se deben utilizar respiradores N95 o aquellos que brinden una protección mayor en casos de procedimientos productores de aerosoles ${ }^{8}$. Los procedimientos que más los ocasionan (pero no limitados a) son intubación y extubación, colocación de sonda intrapleural, broncoscopia, ventilación, traqueostomía, endoscopia, laparoscopia (evacuación de neumoperitoneo o aspiración de líquidos, reanimación cardiopulmonar) y uso de electrocauterio $0^{7,8,10}$.

Debe ser garantizada la seguridad de todo el equipo quirúrgico. El EPP adecuado incluye, además de los respiradores N95, doble par de guantes, lentes con protección lateral ("goggles"), caretas, gorro, botas y bata impermeable para realizar el procedimiento ${ }^{1,9,10}$. Idealmente tanto el vestido como el desvestido 
del EPP debe ser meticuloso y vigilado por una persona entrenada ${ }^{11}$. Los participantes se colocaron bata y guantes estériles sobre el EPP.

Todas las cirugías realizadas en este estudio fueron con anestesia general. En aquellas que sean realizadas con el paciente despierto, éste debe permanecer con respirador N95 para disminuir el riesgo de aerosoles producido por él ${ }^{7}$. En los casos que ameriten o se decida anestesia general, la inducción anestésica debe ser de preferencia de secuencia rápida e intubación ${ }^{7,10}$. Los cirujanos y el resto del equipo quirúrgico no necesario para la intubación deben permanecer fuera de sala hasta que el procedimiento de inducción e intubación haya finalizado. De preferencia esperar un periodo aproximado de 3 minutos para permitir el intercambio de aire en la sala ${ }^{6,8,9}$. El personal que ingresa al quirófano debe ser el menor posible, sin observadores o visitantes y las puertas deben mantenerse cerradas. Una vez dentro no deben de salir hasta que la cirugía termine y una vez fuera no deben volver a entrar $1,7,8$.

Con respecto a las cirugías que realizamos, involucran a diferentes servicios quirúrgicos, tanto de procedimientos electivos como urgentes. El abordaje fue el que se consideró ideal para el tratamiento del padecimiento de acuerdo con la enfermedad y al servicio tratante ${ }^{11}$.

Al momento de planear la intervención, el cirujano debe priorizar abordajes que disminuyan la exposición del equipo quirúrgico y la duración de la cirugía ${ }^{5}$. Algunas consideraciones relacionadas con nuestro grupo quirúrgico. Al ser un padecimiento con afectación principalmente respiratoria, era de esperar una gran frecuencia de traqueostomías (que representaron más de una tercera parte de las cirugías) debido a la necesidad de apoyo mecánico ventilatorio prolongado en muchos pacientes. El tiempo recomendado para realizarla no está todavía determinado. Con grupos que refieren intervención temprana (menor de 7 días) y otros tiempos mayores ${ }^{12}$. La decisión de nuestro hospital es realizarla posterior a dos a tres semanas, esto derivado de la posibilidad de una mejoría en la evolución de los pacientes y con esto retiro de la ventilación mecánica y una disminución de la carga viral de los pacientes con esta espera. Otro aspecto es relativo a los procedimientos de cirugía general, nuestro segundo grupo más grande de pacientes. Algunos autores prefieren evitar abordaje laparoscópico y realizar cirugía abierta o convencional por cuestiones de disminuir el tiempo quirúrgico y el riesgo de aerosoles ${ }^{1,8}$. En caso de preferir cirugía laparoscópica habrá que tener cuidado al momento de aspirar líquidos durante la cirugía y en los momentos que se realiza drenaje del neumoperitoneo, siendo esto preferible a través de un sistema cerrado o con la colocación de un filtro. En nuestros pacientes, se decidió manejo abierto en todos. El uso de electrocauterio se recomienda con la mínima intensidad posible, e incluso evitarlo, para evitar la formación de aerosoles ${ }^{1,9}$.

Al término de la cirugía, es recomendado recuperar al paciente en sala y llevarlo directamente a su cama en el área COVID o a la terapia intensiva. El personal de traslado debe utilizar el EPP correspondiente y no debe ser el mismo del procedimiento quirúrgico. El personal que intervino en la cirugía debe cambiarse completamente de ropa e incluso bañarse, y se recomienda retirarse del hospital ${ }^{8}$.

El seguimiento postoperatorio es el habitual, sin embargo, muchos pacientes hospitalizados por COVID presentan comorbilidades y la mortalidad puede ser mayor derivado de esto ${ }^{1} .^{[1]}$ Ya que muchos de nuestros pacientes ya presentaban manifestaciones respiratorias previo a la cirugía, es muy difícil diferenciar si la evolución posoperatoria de los pacientes en este aspecto fue secundario a la enfermedad quirúrgica o al COVID-19. Sin embargo, un estudio reportó complicaciones respiratorias posoperatorias en el $28 \%$ de los pacientes con COVID-19 comparado con un histórico de $8 \%$ de pacientes posoperatorios ${ }^{13}$. Una tercera parte de nuestros pacientes fueron sometidos a traqueostomía (que fue considerada como cirugía electiva) por lo que no es posible tampoco realizar una asociación entre evolución y cirugía programada y electiva ${ }^{13}$.

La mortalidad general en pacientes sometidos a tratamiento quirúrgico presentada en nuestra unidad es de $26 \%$, es similar a la referida en la serie más grande reportada hasta el momento de pacientes sometidos a cirugía ${ }^{13}$.

\section{Conclusión}

Los pacientes con COVID-19 pueden requerir tratamiento quirúrgico. Es necesario tener un protocolo establecido sobre el mejor manejo de estos pacientes de acuerdo a las características del hospital y la infraestructura. EI EPP es fundamental para el personal de salud involucrado en el tratamiento de los pacientes y con esto disminuir el riesgo de infección. Este estudio incluye a los pacientes que se someten a cualquier procedimiento quirúrgico, lo cual generaliza los resultados. Los pacientes con COVID-19 presentan frecuentemente comorbilidades y complicaciones respiratorias que aumentan la mortalidad posoperatoria, 
por lo que el criterio del cirujano sobre la necesidad de la cirugía es muy importante.

\section{Conflicto de intereses}

Los autores declaran no tener ningún conflicto de intereses con respecto a este trabajo.

\section{Responsabilidades éticas}

Protección de personas y animales. Los autores declaran que para esta investigación no se han realizado experimentos en seres humanos ni en animales.

Confidencialidad de los datos. Los autores declaran que han seguido los protocolos de su centro de trabajo sobre la publicación de datos de pacientes.

Derecho a la privacidad y consentimiento informado. Los autores han obtenido el consentimiento informado de los pacientes y/o sujetos referidos en el artículo. Este documento obra en poder del autor de correspondencia.

\section{Bibliografía}

1. Gao Y, Xi H, Chen L. Emergency Surgery in Suspected COVID-19 Patients with Acute Abdomen: Case Series and Perspectives. Ann Surg $2020 ; 1$.
2. guidance for triage of nonemergent_surgical_procedures general surgery.pdf [Internet]. [citado 2020 may 19];Available from: https://www. facs.org/-/media/files/covid19/guidance_for_triage_of_nonemergent_surgical procedures general surgery.ashx

3. Al-Jabir A, Kerwan A, Nicola M, Alsafi Z, Khan M, Sohrabi C, et al. Impact of the Coronavirus (COVID-19) pandemic on surgical practice - Part 1. Int J Surg 2020;79:168-79.

4. Flemming S, Hankir M, Ernestus R-I, Seyfried F, Germer C-T, Meybohm P, et al. Surgery in times of COVID-19-recommendations for hospital and patient management. Langenbecks Arch Surg [Internet] 2020 [citado 2020 may 19]; Available from: http://link.springer. com/10.1007/s00423-020-01888-x

5. Spolverato G, Capelli G, Restivo A, Bao QR, Pucciarelli S, Pawlik TM, et al. The management of surgical patients during the coronavirus disease 2019 (COVID-19) pandemic. Surgery 2020;168(1):4-10.

6. Yeo D, Yeo C, Kaushal S, Tan G. COVID-19 and the General Surgical Department - Measures to Reduce Spread of SARS-COV-2 Among Surgeons: Ann Surg 2020;1.

7. Coccolini F, Perrone G, Chiarugi M, Di Marzo F, Ansaloni L, Scandroglio I, et al. Surgery in COVID-19 patients: operational directives. World $J$ Emerg Surg 2020;15(1):25.

8. considerations optimum surgeon protection.pdf [Internet]. [citado 2020 may 19];Available from: https://www.facs.org/-/media/files/covid19/considerations_optimum_surgeon_protection.ashx

9. Orthopoulos G, Fernandez GL, Dahle JL, Casey E, Jabbour N. Perioperative Considerations During Emergency General Surgery in the Era of COVID-19: A U.S. Experience. J Laparoendosc Adv Surg Tech 2020;30(5):481-4.

10. Al-Balas M, Al-Balas HI, Al-Balas H. Surgery during the COVID-19 pandemic: A comprehensive overview and perioperative care. Am J Surg 2020;S0002961020302221.

11. Balibrea JMose, Badia JMose, Rubio Pérez I, Martín Antona E, Álvarez Peña E, García Botella S, et al. Manejo quirúrgico de pacientes con infección por COVID-19. Recomendaciones de la Asociación Española de Cirujanos. Cir Esp 2020;98(5):251-9.

12. Chiesa-Estomba CM, Lechien JR, Calvo-Henríquez C, Fakhry N, Karkos PD, Peer S, et al. Systematic review of international guidelines for tracheostomy in COVID-19 patients. Oral Oncol 2020;108:104844.

13. Nepogodiev D, Bhangu A, Glasbey JC, Li E, Omar OM, Simoes JF, et al. Mortality and pulmonary complications in patients undergoing surgery with perioperative SARS-CoV-2 infection: an international cohort study. The Lancet 2020;396(10243):27-38. 